\title{
Infratentorial pathology in frontotemporal dementia: cerebellar grey and white matter alterations in FTD phenotypes
}

\author{
Mary Clare McKenna ${ }^{1} \cdot$ Rangariroyashe H. Chipika $^{1} \cdot$ Stacey Li Hi Shing ${ }^{1} \cdot$ Foteini Christidi $^{1}$. Jasmin Lope ${ }^{1}$. \\ Mark A. Doherty ${ }^{2} \cdot$ Jennifer C. Hengeveld ${ }^{2} \cdot$ Alice Vajda $^{2} \cdot$ Russell L. McLaughlin $^{2} \cdot$ Orla Hardiman $^{1}$. \\ Siobhan Hutchinson ${ }^{3} \cdot$ Peter Bede ${ }^{1,3}$
}

Received: 12 February 2021 / Revised: 19 April 2021 / Accepted: 19 April 2021 / Published online: 13 May 2021

(c) The Author(s) 2021

\begin{abstract}
The contribution of cerebellar pathology to cognitive and behavioural manifestations is increasingly recognised, but the cerebellar profiles of FTD phenotypes are relatively poorly characterised. A prospective, single-centre imaging study has been undertaken with a high-resolution structural and diffusion tensor protocol to systematically evaluate cerebellar grey and white matter alterations in behavioural-variant FTD(bvFTD), non-fluent variant primary progressive aphasia(nfvPPA), semantic-variant primary progressive aphasia(svPPA), C9orf72-positive ALS-FTD(C9 + ALSFTD) and C9orf72-negative ALS-FTD(C9-ALSFTD). Cerebellar cortical thickness and complementary morphometric analyses were carried out to appraise atrophy patterns controlling for demographic variables. White matter integrity was assessed in a study-specific white matter skeleton, evaluating three diffusivity metrics: fractional anisotropy (FA), axial diffusivity (AD) and radial diffusivity (RD). Significant cortical thickness reductions were identified in: lobule VII and crus I in bvFTD; lobule VI VII, crus I and II in nfvPPA; and lobule VII, crus I and II in svPPA; lobule IV, VI, VII and Crus I and II in C9 + ALSFTD. Morphometry revealed volume reductions in lobule V in all groups; in addition to lobule VIII in C9+ ALSFTD; lobule VI, VIII and vermis in C9-ALSFTD; lobule V, VII and vermis in bvFTD; and lobule V, VI, VIII and vermis in nfvPPA. Widespread white matter alterations were demonstrated by significant fractional anisotropy, axial diffusivity and radial diffusivity changes in each FTD phenotype that were more focal in those with C9+ ALSFTD and svPPA. Our findings indicate that FTD subtypes are associated with phenotype-specific cerebellar signatures with the selective involvement of specific lobules instead of global cerebellar atrophy.
\end{abstract}

Keywords Frontotemporal dementia $\cdot$ Cerebellum $\cdot$ PPA $\cdot$ Behaviour $\cdot$ MRI $\cdot$ Cortical thickness

\section{Introduction}

The function of the cerebellum continues to be defined, particularly with respect to its physiological role in cognition and behaviour. Clinical observations from acquired cerebellar pathologies have consistently highlighted the posterior

Peter Bede

bedep@tcd.ie

1 Computational Neuroimaging Group, Trinity Biomedical Sciences Institute, Trinity College Dublin, Peter Bede, Room 5.43, Pearse Street, Dublin 2, Ireland

2 Complex Trait Genomics Laboratory, Smurfit Institute of Genetics, Trinity College Dublin, Dublin 2, Ireland

3 Department of Neurology, St James's Hospital, Dublin, Ireland predominance of cognitive functioning in the cerebellum $[1,2]$ and imaging studies have confirmed the specific role of lobules VI, VIIA, VIIB, IX and crus I/II in mediating cognitive processes [3-5]. Posterior cerebellar injuries may manifest in multi-domain cognitive deficits including verbal memory, language, visuospatial, executive function and sequencing abilities; while cognition may be relatively preserved in those with anterior cerebellar insults [6]. Cerebellar pathology may contribute to impairments in social cognition [7], language deficits [8] and pathological crying and laughing $[9,10]$. Lesions of the vermis have been linked to emotional dysregulation such as irritability, impulsivity and disinhibition [11]. While the neuropsychological sequelae of acute vascular, neoplastic and inflammatory cerebellar pathologies are widely recognised, cognitive deficits associated with slowly progressive neurodegenerative 
conditions are less well characterised. There is a striking paucity of imaging data on cerebellar involvement in FTD [12-14] despite ample post-mortem evidence of cerebellar pathology [15]. A recent meta-analysis noted lobule VI, VIIb, VIIIb atrophy in bvFTD, crus I and lobule VI volume loss in svPPA [16]. Genetic FTD subtypes appear to exhibit specific grey matter cerebellar abnormalities [12-14, 17, 18]. The C9orf72 genotype has been linked to focal crus I and lobule VIIa degeneration, MAPT mutation associated with vermis pathology, and GRN mutation with relatively preserved cerebellar integrity [13]. Interestingly, regional cerebellar atrophy was detected in asymptomatic $C 9$ orf 72 mutation carriers [19]. ALS-FTD has been linked to superior (lobules I-VI), crus and vermis degeneration [20]. Other cerebellar regions, such as the cerebellar crura and lobule VI may be involved in all FTD subtypes [16]. This region is often labelled 'the cognitive cerebellum' because of its central role in cognitive processing; the extent of atrophy in this area is thought to correlate with cognitive performance across a multitude of domains [14, 16]. Existing studies suggest that cerebellar abnormalities are most widespread in those with ALS-FTD and bvFTD, and may be relatively focal in those with svPPA or nfvPPA [14, 17, 20]. Selective cerebellar atrophy seems to mirror patterns of cerebral cortical pathology [21,22] and is likely to be defined by cerebello-cerebral connectivity. These observations further support the 'dysmetria of thought theory' whereby cerebellar lesions result in individual patterns of cognitive dysfunction dependent on the cortico-cerebellar tracts involved [23]. The majority of cerebellar imaging studies in FTD solely appraise grey matter alterations, white matter degeneration is less well characterised in vivo, and there is a lack of cerebellar functional and metabolic studies. Cerebellar hypometabolism have been reported [16, 24, 25] but the majority of PET studies focus on supratentorial regions.

Post-mortem studies in FTD also disproportionately focus on supratentorial regions. Much of the limited post-mortem data of cerebellar pathology in FTD pertains to a select cohort of those carrying the C9orf72 mutation. In such cases, TDP-43 negative, ubiquitin and p62-positive neuronal cytoplasmic inclusions were noted in the granular layer of the cerebellar cortex, but these findings are not exclusive to this genotype $[15,26,27]$. Cerebellar atrophy has been described in those carrying the C9orf72 gene mutation, but not in those carrying the MAPT mutation [26, 28]. A case series of two sisters with a clinical diagnosis of bvFTD and no established genetic mutation, demonstrated abundant abnormal tau deposition in the cerebellum, with a distinctly different morphology from the more common tauopathies [29].

Emerging imaging and post-mortem data lends credence to the body of evidence that cerebellar involvement may contribute to the clinical manifestations of FTD. These observations provide the rationale to characterise cerebellar signatures in FTD phenotypes using a multiparametric grey and white matter imaging protocol. The main objective of this FTD study is to ascertain if focal cerebellar degeneration may be identified in vivo, and establish phenotype-specific and overlapping radiological features.

\section{Methods}

\section{Participants}

A total of 156 participants were included in a prospective imaging study of frontotemporal dementia; 7 patients with behavioural-variant FTD ('bvFTD', mean age 60.71 \pm 3.3 ), 12 patients with non-fluent-variant primary progressive aphasia ('nfvPPA', mean age $61.5 \pm 2.96$ ), 3 patients with semantic-variant primary progressive aphasia ('svPPA', mean age $61.66 \pm 6.42$ ), 12 ALS-FTD patients carrying C9orf72 GGGGCC hexanucleotide repeat expansions ('C9 + ALSFTD', mean age 58.65 111.22), 12 C9orf72negative ALS-FTD patients repeats ('C9-ALSFTD', mean age $59.95 \pm 7.67$ ), and 110 healthy controls ('HC', mean age $59.21 \pm 10.5)$. All participants provided informed consent in accordance with the Medical Ethics Approval of the research project (Beaumont Hospital, Dublin, Ireland). Exclusion criteria included prior traumatic brain injury, cerebrovascular events, comorbid neoplastic, paraneoplastic, or neuroinflammatory diagnoses. FTD and ALS-FTD was diagnosed based on the Rascovsky criteria [30] and participating ALS patients had 'probable' or 'definite' ALS according to the revised El Escorial research criteria. Healthy controls were unrelated to patients and had no known family history of neurodegenerative conditions.

\section{Magnetic resonance imaging}

Imaging data were acquired on a $3 \mathrm{~T}$ Philips Achieva Magnetic resonance (MR) platform with an 8-channel receiveonly head coil. The standardised imaging protocol included a high-resolution $\mathrm{T}_{1}$-weighted $(\mathrm{T} 1 \mathrm{w})$ and a 32-direasction diffusion tensor imaging (DTI). T1w was acquired with a 3D Inversion Recovery prepared Spoiled Gradient Recalled echo (IR-SPGR) sequence with the following parameters; field-ofview (FOV) of $256 \times 256 \times 160 \mathrm{~mm}$, flip angle $=8^{\circ}$, spatial resolution of $1 \mathrm{~mm}^{3}$, SENSE factor $=1.5, \mathrm{TR} / \mathrm{TE}=8.5 / 3.9$ $\mathrm{ms}, \mathrm{TI}=1060 \mathrm{~ms}$. DTI data were acquired with a spinecho echo planar imaging (SE-EPI) pulse sequence using a 32-direction Stejskal-Tanner diffusion encoding scheme, FOV $=245 \times 245 \times 150 \mathrm{~mm}, 60$ slices with no interslice gap, spatial resolution $=2.5 \mathrm{~mm}^{3}$, TR/TE $=7639 / 59 \mathrm{~ms}$, SENSE factor $=2.5, b$ values $=0,1100 \mathrm{~s} / \mathrm{mm}^{2}$, dynamic stabilisation 
and spectral presaturation with inversion recovery (SPIR) fat suppression.

\section{Morphometry}

First, total intracranial volumes (TIV) were estimated for each subject to be used as a covariate in subsequent region of interest (ROI) morphometric analyses. As described previously [31, 32], TIV estimation was performed by linearly aligning each participant's skull-stripped brain image to the MNI152 standard, and the inverse of the determinant of the affine registration matrix was calculated and multiplied by the size of the template. FMRIB's FSL-FLIRT was used for spatial registration and FSL-FAST for tissue-type segmentation. Partial grey matter, white matter, and CSF volumes were added for TIV estimation. Grey matter pathology in the FTD groups was evaluated by ROI morphometry using FMRIB's FSL suite. Pre-processing steps included skull removal (BET), motion corrections and tissue-type segmentation [33]. Grey-matter partial volume images were aligned to the MNI152 standard space using affine registration. A study-specific grey matter template was created representing each study group to which the grey matter images of each participant were subsequently non-linearly co-registered. Permutation-based non-parametric inference was utilised to contrast each patient group with healthy control implementing the threshold-free cluster enhancement (TFCE) method. Design matrices included group membership, age, sex and TIV [34]. Statistics were restricted to a cerebellar ROI mask defined by label 1 of the MNI structural atlas. Resulting statistical maps were thresholded at $p<0.05$ and visualised in FSLeyes. The aid the localisation of statistically significant clusters the Diedrichsen probabilistic atlas was used as undelay [35].

\section{Cortical thickness analyses}

To evaluate cerebellar cortical thickness alterations, the cerebellum was segmented using a validated parcellation algorithm. [36-38] A patch-based segmentation algorithm was then applied to obtain cerebellar GM metrics for each lobule, separately for the right and left cerebellar hemispheres [36]. As a quality-control step, anatomical parcellation and tissuetype segmentation was individually verified for each subject. The following labels were used to retrieve regional cortical thickness values: lobules I-V, lobule VI, lobule VIIb, lobules VIII-X, Crus I, and Crus II. To test the effect of group membership on cerebellar cortical thickness in each lobule, Multivariate analyses of covariance (MANCOVAs) were conducted for the right and left cerebellar hemispheres separately, designating lobular cortical thickness as dependent variable, group membership as independent factor and age and gender as covariates. In case of a significant multivariate omnibus test, post hoc comparisons were considered significant at $p<0.05$, following false-discovery rate (FDR) corrections for multiple comparisons to reduce Type I error.

\section{White matter analyses}

Raw DTI data underwent eddy current corrections and skull removal before a tensor model was fitted to generate maps of fractional anisotropy (FA), axial diffusivity (AD), and radial diffusivity (RD). The tract-based statistics (TBSS) module of FMRIB's software library was utilised for nonlinear registration and skeletonisation of individual DTI images. A mean FA mask was created and each subject's individual $\mathrm{AD}, \mathrm{FA}$ and $\mathrm{RD}$ images were merged into fourdimentional (4D) AD, FA and RD image files. The input file order matched the group membership variables in the design matrix. Permutation-based non-parametric inference was used for the two-way, voxelwise comparison of diffusivity parameters between each FTD group and controls using design matrix-defined contrasts which included age and gender as covariates. The study-specific white matter skeleton was masked by atlas-defined labels for the entire cerebellum (left and right hemispheres) to restrict analyses to the cerebellum. The threshold-free cluster enhancement (TFCE) method was applied and results considered significant at a $p<0.01$ TFCE family-wise error (FWE).

\section{Genetic testing}

Pathogenic GGGGCC hexanucleotide repeat expansions in $C 9$ orf 72 were screened for with repeat-primed PCR as described previously [39, 40]. Amplified DNA fragments were evaluated with the Applied Biosystems 3130xl Genetic Analyser (Foster City, CA, USA) and visualised using GeneMapper version 4.0. GGGGCC hexanucleotide repeat expansions longer than 30 were considered positive. Participating patients were also screened and tested negative for other mutations associated with ALS and FTD: SOD1, ALS2, SETX, SPG11, FUS, VAPB, ANG, TARDBP, FIG4, OPTN, ATXN2, VCP, UBQLN2, SIGMAR1, CHMP2B, PFN1, ERBB4, HNRNPA1, MATR3, CHCHD10, UNC13A, DAO, DCTN1, NEFH, PRPH, SQSTM1, TAF15, SPAST, ELP3, LMNB1, SARM1, C21orf2, NEK1, FUS, CHMP2B, GRN, MAPT, PSEN1, PSEN2, TBK1.

\section{Results}

\section{Morphometry}

Region-of-interest morphometry in a study-specific, atlasdefined cerebellar grey matter mask revealed phenotypespecific patterns of atrophy at $p<0.05$ TFCE (corrected for 
age, sex and TIV). GGGGCC hexanucleotide repeat carrying ALS-FTD patients exhibited symmetric lobule VIII and lobule V atrophy. C9orf72-negative ALS-FTD patients displayed lobule V, VI, VIII and vermis atrophy. Behaviouralvariant FTD patients showed vermis, lobule V, lobule VII and symmetric posterior-inferior volume reductions. Nonfluent variant primary progressive aphasia patients exhibited widespread atrophy including lobules V, VI, VIII, and the vermis. Semantic-variant FTD patients displayed volume loss in crus I, Crus II, and lobule V on the left (Fig. 1.)

\section{Cortical thickness}

The evaluation of cortical thickness profiles revealed the preferential involvement of specific cerebellar lobules in FTD phenotypes with the apparent sparing of other cerebellar regions.

Following FDR corrections and statistical adjustments for demographic factors, C9orf72-positive ALS-FTD patients exhibited reduced cortical thickness in Lobule IV, VI,VIIb, Crus I and II. Crus II and lobule VI was affected in both cerebellar hemispheres (Table 1). Cortical thinning did not reach statistical significance in C9orf72-negative ALS-FTD patients in any of the evaluated cerebellar regions. Patients with behavioural-variant FTD showed cortical thinning in crus I and a trend of thinning post FDR in lobule VII of the right cerebellar hemisphere. Patients with non-fluent variant primary progressive aphasia (nfvPPA) exhibited lobule VI, VIIb, crus I and II. Lobule VI and crus II atrophy was observed in each hemisphere. Patients with semantic-variant FTD (svPPA) showed lobule VIIb, crus I and II degeneration in the right cerebellar hemisphere.

\section{White matter alterations}

Permutation-based non-parametric statistics confirmed focal diffusivity alterations at $p<0.01$ TFCE (corrected for age and sex) in a study-specific cerebellar white matter skeleton. Reduced fractional anisotropy, reduced axial diffusivity and increased radial diffusivity were detected in each FTD phenotype with reference to healthy controls. Patterns of white matter vulnerability varied along the ALS-FTD spectrum (Fig. 2). C9orf72-positive ALS-FTD patients exhibited reduced FA in the superior cerebellar peduncle, reduced $\mathrm{AD}$ in Crus I and II, and increased RD in lobules I-IV as well as in the superior peduncle. C9orf72-negative ALS-FTD patients displayed widespread, symmetric, multilobular FA reductions, focal $\mathrm{AD}$ reduction in the right lobule $\mathrm{V}$, and increased RD in crus I and II in the right cerebellar hemisphere. Patients with behavioural-variant FTD showed
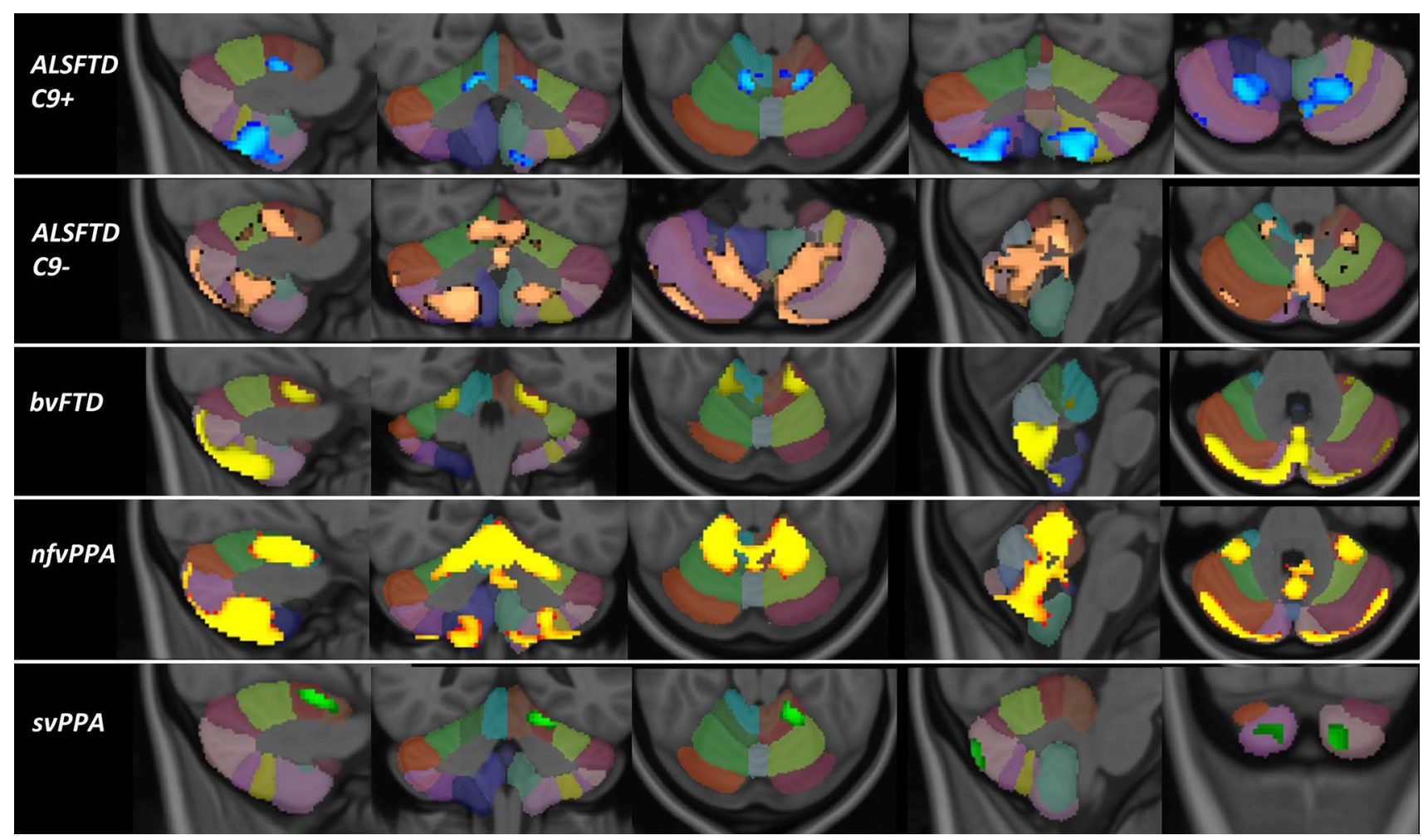

Fig. 1 Cerebellar grey matter changes in FTD phenotypes at $p<0.05$ TFCE corrected for age, gender and TIV. Focal changes in C9+ALSFTD are indicated in blue, C9-ALSFTD in copper colour, bvFTD in yellow, nfvPPA red-yellow, svPPA in green. The Diedrichsen probabilistic cerebellar atlas is presented as underlay to aid localisation 


\begin{tabular}{|c|c|c|c|c|c|c|c|c|c|c|c|c|c|}
\hline & 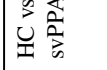 & તุ. & 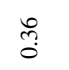 & on & $\hat{o}$ & ò & $\stackrel{f}{f}$ & ot & oे & oे & $\vec{n}$ & กิ & ồ \\
\hline & 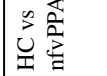 & $\overrightarrow{\widehat{T}}$ & $\dot{o}$ & $\stackrel{\substack{0 \\
\infty}}{\infty}$ & तె & $\stackrel{0}{\circ}$ & ஜ̊ํ: & 离 & $\vec{د}$ & f̊ & in & 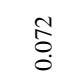 & $\stackrel{n}{0}$ \\
\hline & 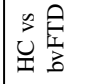 & d & f. & $\stackrel{m}{:}$ & तి & ֻั & ֻัવ & $\stackrel{\substack{\infty \\
0}}{0}$ & m. & ఫొ & $\stackrel{\infty}{\infty}$ & సิ & ò \\
\hline & 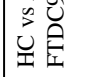 & $\stackrel{\circ}{0}$ & 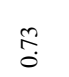 & $\stackrel{5}{\circ}$ & 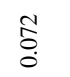 & $\stackrel{\text { on }}{\stackrel{8}{0}}$ & $\begin{array}{l}\text { Do } \\
0 \\
0\end{array}$ & : & fे & స్తి & $\dot{o}$ & 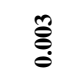 & 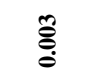 \\
\hline & 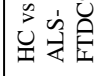 & $\stackrel{n}{3}$ & ֻั & $\stackrel{\substack{0 \\
0}}{0}$ & 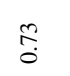 & $\frac{n}{0}$ & $\overrightarrow{\overrightarrow{0}}$ & aे & $\overrightarrow{\stackrel{フ}{0}}$ & $\begin{array}{l}\stackrel{0}{\infty} \\
\stackrel{0}{\circ}\end{array}$ & กุ & $\begin{array}{l}f \\
0\end{array}$ & $\stackrel{f}{0}$ \\
\hline & 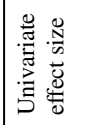 & 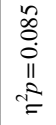 & 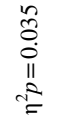 & 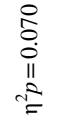 & $\begin{array}{l}8 \\
0 \\
0 \\
11 \\
\cong \\
=\end{array}$ & 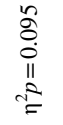 & $\begin{array}{l}\infty \\
\stackrel{\infty}{0} \\
0 \\
\stackrel{0}{11} \\
\cong\end{array}$ & 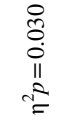 & $\begin{array}{l}\because 0 \\
0 \\
0 \\
11 \\
\cong\end{array}$ & $\begin{array}{l}\text { ơ } \\
0 \\
0 \\
11 \\
\cong\end{array}$ & $\begin{array}{l}\vec{J} \\
0 \\
\text { II } \\
\stackrel{2}{\rightleftharpoons}\end{array}$ & $\begin{array}{l} \pm \\
0 \\
I 1 \\
\cong \\
=\end{array}$ & $\begin{array}{l}\stackrel{\infty}{+} \\
\stackrel{0}{0} \\
\cong \\
\cong\end{array}$ \\
\hline 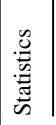 & 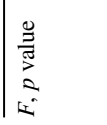 & is & & & & & & & & & & & 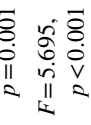 \\
\hline & 育 & 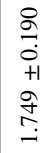 & 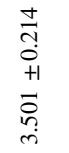 & $\begin{array}{l}+ \\
\stackrel{+}{0} \\
\dot{+} \\
+1 \\
\stackrel{+}{a} \\
\overrightarrow{+}\end{array}$ & 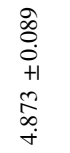 & 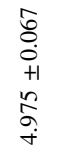 & $\begin{array}{l}2 \\
a \\
0 \\
+1 \\
+1 \\
5 \\
+ \\
\dot{+}\end{array}$ & $\begin{array}{l}\hat{0} \\
0 \\
+ \\
+1 \\
\hat{0} \\
+\end{array}$ & $\begin{array}{l}\circ \\
\circ \\
0 \\
+1 \\
\infty \\
\infty \\
\stackrel{+}{+}\end{array}$ & 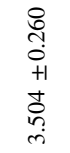 & 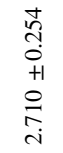 & 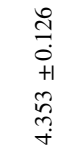 & 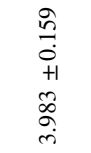 \\
\hline & $\begin{array}{l}\text { 育 } \\
\text { 彦 }\end{array}$ & $\begin{array}{l}n \\
o \\
0 \\
+1 \\
+1 \\
\text { to } \\
\stackrel{-}{+}\end{array}$ & $\begin{array}{l}\hat{0} \\
0 \\
0 \\
+1 \\
\infty \\
\infty \\
\dot{m}\end{array}$ & $\begin{array}{l}\tilde{J} \\
\stackrel{0}{0} \\
\dot{+} \\
+1 \\
\vec{a} \\
\overrightarrow{+} \\
+\end{array}$ & 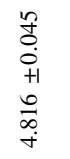 & $\begin{array}{l}+ \\
0 \\
0 \\
\stackrel{+}{+} \\
+1 \\
\infty \\
\infty \\
\dot{+}\end{array}$ & $\begin{array}{l}\mathscr{8} \\
\stackrel{0}{+} \\
\dot{+} \\
+1 \\
\infty \\
\stackrel{+}{+} \\
\dot{+}\end{array}$ & $\begin{array}{l}n \\
\hat{o} \\
\dot{+} \\
+1 \\
\infty \\
o \\
o \\
+\end{array}$ & $\begin{array}{l}\infty \\
\stackrel{0}{0} \\
\dot{+} \\
+1 \\
\underset{+}{+}\end{array}$ & $\begin{array}{l}0 \\
- \\
0 \\
+1 \\
n \\
\stackrel{n}{r} \\
i\end{array}$ & $\begin{array}{l}\hat{I} \\
0 \\
+ \\
+1 \\
o \\
\stackrel{0}{i} \\
\text { i }\end{array}$ & $\begin{array}{l}8 \\
\stackrel{0}{0} \\
0 \\
+1 \\
+ \\
o \\
+ \\
\dot{\sigma}\end{array}$ & $\begin{array}{l}8 \\
\& \\
0 \\
+1 \\
+1 \\
\& \\
\stackrel{0}{+} \\
+\end{array}$ \\
\hline 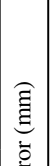 & $\begin{array}{l}\text { 屋 } \\
\text { 咅 }\end{array}$ & 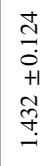 & $\begin{array}{l}\stackrel{q}{+} \\
\dot{0} \\
+1 \\
+\infty \\
\infty \\
o \\
\dot{r}\end{array}$ & $\begin{array}{l}n \\
\hat{o} \\
\dot{0} \\
+1 \\
\infty \\
\infty \\
\stackrel{0}{+} \\
+\end{array}$ & $\begin{array}{l}\infty \\
0 \\
0 \\
\dot{0} \\
+1 \\
\stackrel{2}{0} \\
\stackrel{2}{+} \\
\dot{+}\end{array}$ & $\begin{array}{l}\text { 志 } \\
\dot{0} \\
+1 \\
+ \\
\dot{a} \\
\dot{\sigma}\end{array}$ & 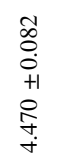 & 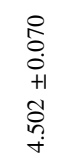 & 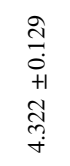 & 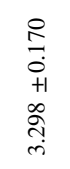 & 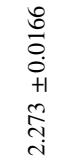 & 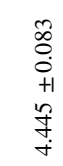 & 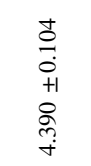 \\
\hline 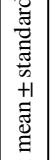 & 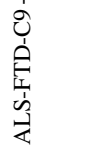 & 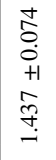 & $\begin{array}{l}0 \\
0 \\
0 \\
+1 \\
+1 \\
\stackrel{0}{0} \\
\tilde{n}\end{array}$ & $\begin{array}{l}\infty \\
\stackrel{0}{0} \\
\dot{+} \\
+1 \\
\hat{\infty} \\
\dot{+} \\
\dot{+}\end{array}$ & $\begin{array}{l}\stackrel{+}{0} \\
\stackrel{0}{0} \\
\dot{+} \\
+1 \\
0 \\
0 \\
\dot{+} \\
\dot{+}\end{array}$ & $\begin{array}{l}\stackrel{0}{0} \\
0 \\
0 \\
+1 \\
\infty \\
\infty \\
\dot{+} \\
\dot{+}\end{array}$ & 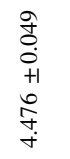 & $\begin{array}{l}\vec{F} \\
\dot{0} \\
+1 \\
+1 \\
? \\
\stackrel{+}{+}\end{array}$ & $\begin{array}{l}\stackrel{0}{0} \\
0 \\
0 \\
+1 \\
0 \\
\stackrel{9}{+}\end{array}$ & $\begin{array}{l}\overrightarrow{0} \\
0 \\
0 \\
+1 \\
\infty \\
0 \\
m \\
m\end{array}$ & $\begin{array}{l}\infty \\
\stackrel{0}{0} \\
0 \\
+1 \\
+1 \\
\infty \\
\stackrel{+}{+} \\
i\end{array}$ & $\begin{array}{l}\hat{\sigma} \\
0 \\
\dot{0} \\
+1 \\
\text { م } \\
m \\
+\end{array}$ & $\begin{array}{l}\text { ठ̊. } \\
0 \\
+ \\
+1 \\
\hat{o} \\
\dot{+} \\
\dot{+}\end{array}$ \\
\hline 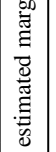 & 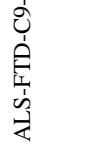 & 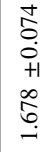 & $\begin{array}{l}0 \\
0 \\
0 \\
+1 \\
+1 \\
n \\
m \\
m \\
m\end{array}$ & $\begin{array}{l}\text { m. } \\
\stackrel{0}{0} \\
+1 \\
\stackrel{0}{0} \\
\stackrel{\sigma}{+}\end{array}$ & 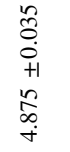 & 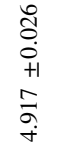 & $\begin{array}{l}\text { ơ } \\
0 \\
+ \\
+1 \\
\stackrel{0}{0} \\
\stackrel{0}{+} \\
\dot{\gamma}\end{array}$ & 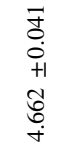 & $\begin{array}{l}0 \\
\vdots \\
0 \\
+1 \\
+1 \\
\vec{b} \\
+\end{array}$ & $\begin{array}{l}\vec{\Xi} \\
\vdots \\
+1 \\
+1 \\
\overrightarrow{0} \\
\dot{n}\end{array}$ & $\begin{array}{l}\text { oे } \\
0 \\
\text { +1 } \\
\text { के } \\
\text { ते }\end{array}$ & 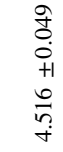 & 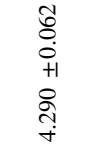 \\
\hline 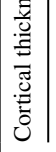 & U. & 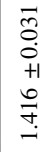 & $\begin{array}{l}\stackrel{n}{0} \\
\stackrel{0}{0} \\
+1 \\
m \\
\vec{n} \\
\tilde{m}\end{array}$ & 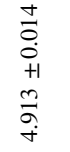 & $\begin{array}{l}n \\
\stackrel{n}{0} \\
0 \\
+1 \\
\infty \\
\infty \\
\infty \\
\dot{+}\end{array}$ & $\begin{array}{l}\vec{\Xi} \\
\dot{0} \\
\dot{+} \\
+1 \\
\infty \\
o \\
\dot{\sigma}\end{array}$ & $\begin{array}{l}\vec{\delta} \\
\dot{+} \\
+1 \\
+ \\
o \\
0 \\
+ \\
+\end{array}$ & $\begin{array}{l}\infty \\
\stackrel{0}{0} \\
\dot{+} \\
+1 \\
\text { ôे } \\
\stackrel{+}{+}\end{array}$ & 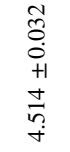 & $\begin{array}{l}\text { mo } \\
0 \\
0 \\
+1 \\
0 \\
i n \\
n \\
n\end{array}$ & $\begin{array}{l}\mathcal{F} \\
\stackrel{0}{0} \\
+1 \\
\vec{G} \\
\vec{j}\end{array}$ & 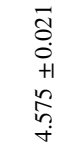 & 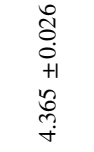 \\
\hline 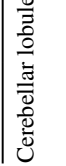 & & $\bar{I}$ & $\exists$ & $z$ & $>$ & 5 & $\stackrel{\rho}{\stackrel{9}{>}}$ & 岁 & 曾 & $\succeq$ & & $\overrightarrow{\underline{u}}$ & 产 \\
\hline
\end{tabular}




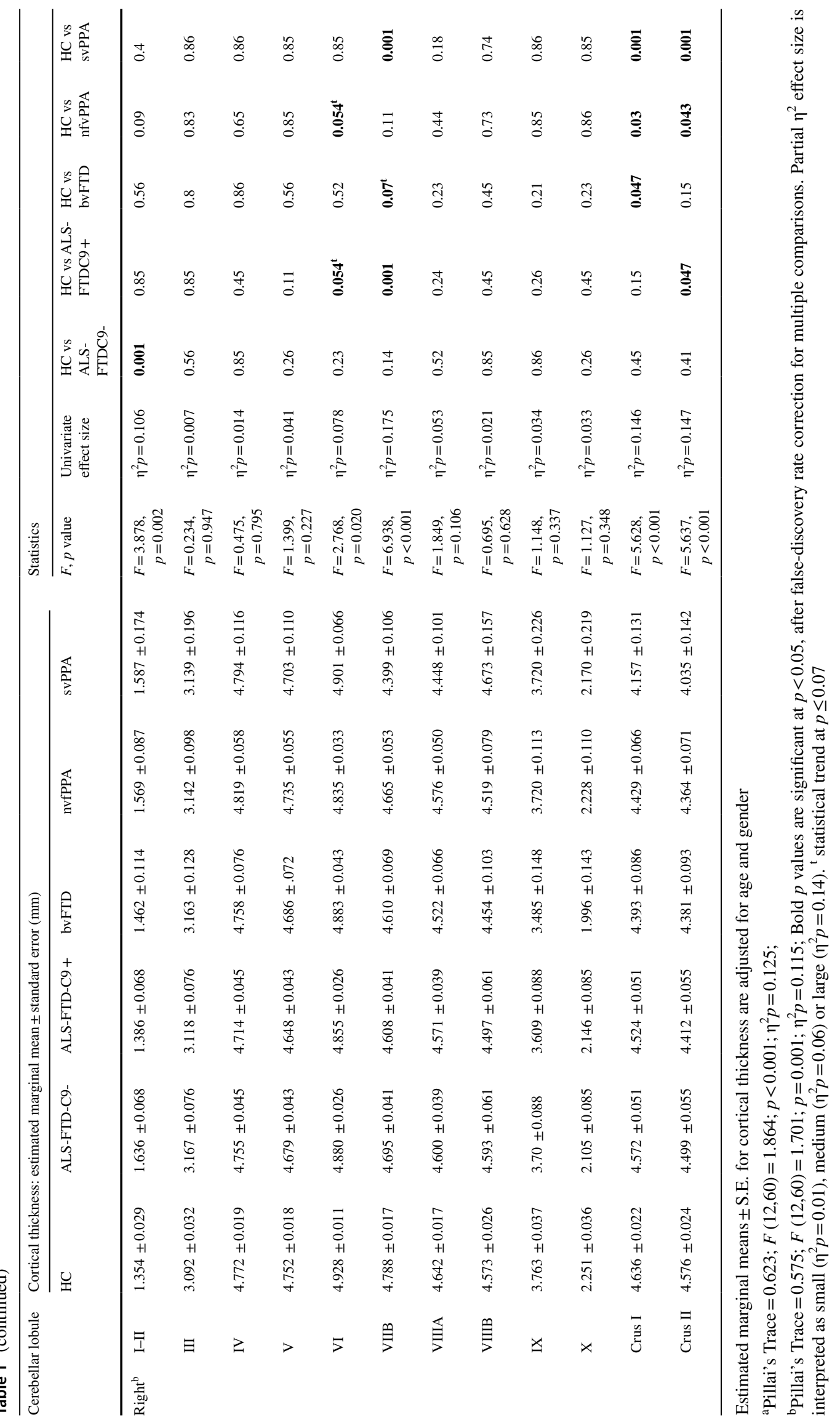




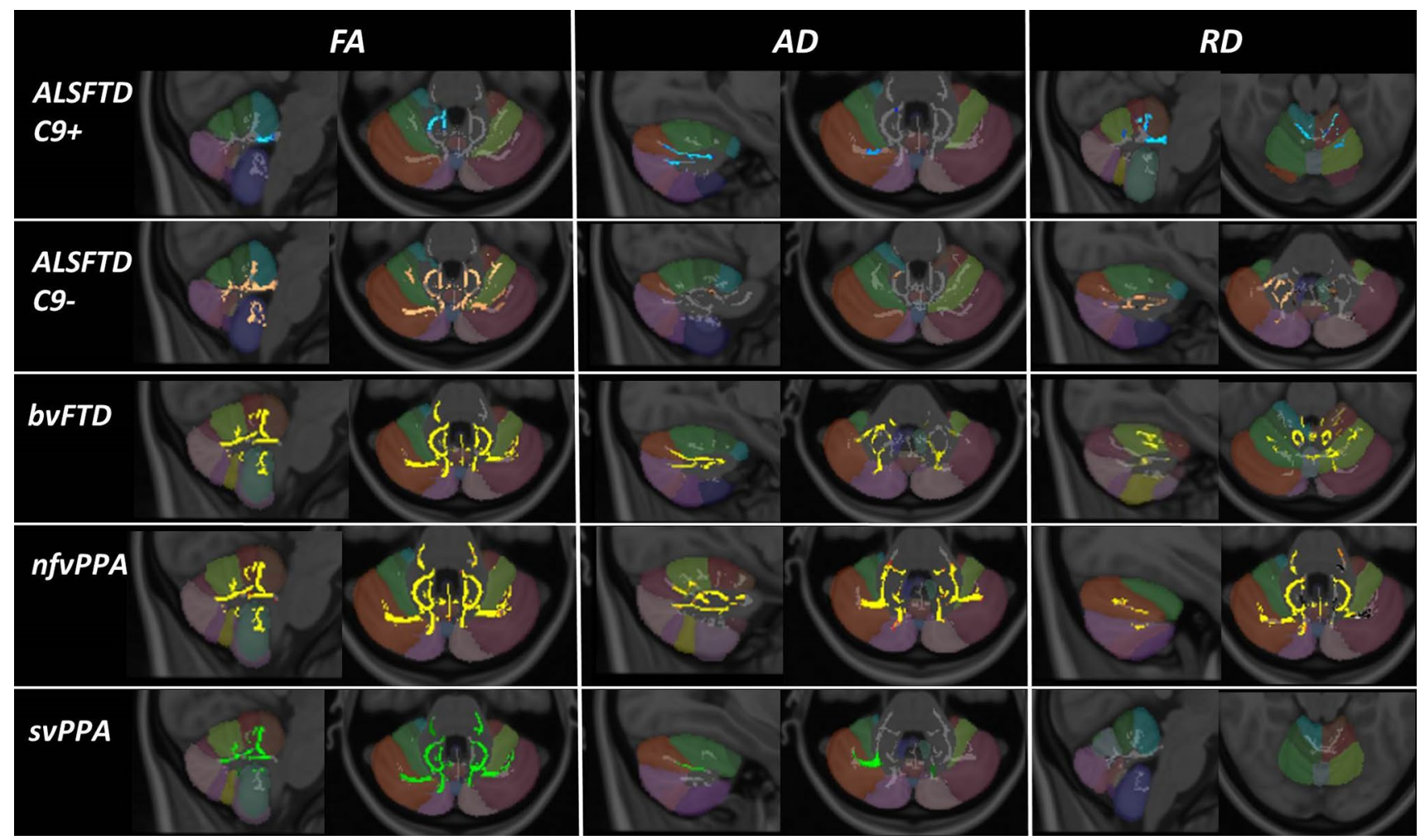

Fig. 2 Tract-based white matter changes in FTD phenotypes as identified by FA, AD and RD alterations at $p<0.01$ TFCE adjusted for age and gender. Changes in C9+ALSFTD are indicated in blue,

FA reductions in nearly the entire cerebellar white matter skeleton, reduced AD in crus I and II, and widespread areas of increased RD in particular in lobule VI. Patients with non-fluent variant primary progressive aphasia (nfvPPA) exhibited multi-lobular FA and AD reductions and similarly widespread RD increases. Patients with semantic-variant FTD (svPPA) showed superior-predominant symmetric FA reductions centred on lobule $\mathrm{V}$, reduced $\mathrm{AD}$ in Crus I, and no $\mathrm{RD}$ alterations at $p<0.01$.
C9-ALSFTD in copper colour, bvFTD in yellow, nfvPPA red-yellow, svPPA in green. The Diedrichsen probabilistic cerebellar atlas is presented as underlay to aid localisation

\section{Summary of findings}

The integration of findings across multiple imaging modalities revealed the selective involvement of cerebellar regions with relatively distinctive imaging signatures along the ALS-FTD spectrum (Table 2.)

Table 2 Summary of focal findings across the five imaging modalities

\begin{tabular}{|c|c|c|c|c|c|}
\hline Study group & Morphometry & FA & $\mathrm{AD}$ & $\mathrm{RD}$ & Cortical thickness \\
\hline C9+ALSFTD & Lobule V, VIII & $\begin{array}{l}\text { Superior cerebellar } \\
\text { peduncle }\end{array}$ & Crus I \& II & $\begin{array}{l}\text { Lobules I-IV superior } \\
\text { peduncle }\end{array}$ & $\begin{array}{l}\text { Lobule IV, VI,VII Crus } \\
\text { I \& II }\end{array}$ \\
\hline C9-ALSFTD & Lobule V, VI, VIII, vermis & Widespread multi-lobular & Lobule V & Crus I \& II & Nil at $p<0.05$ post FDR \\
\hline bvFTD & Lobule V, VII, vermis & widespread multi-lobular & Crus I \& II & Widespread multi-lobular & lobule VII, crus I \\
\hline nfvPPA & Lobule V, VI, VIII, vermis & Widespread multi-lobular & $\begin{array}{l}\text { Widespread } \\
\text { multi- } \\
\text { lobular }\end{array}$ & Widespread multi-lobular & lobule VI, VII, crus I \& II \\
\hline svPPA & Lobule V, crus I \& II & $\begin{array}{l}\text { Lobule V, and superior } \\
\text { cerebellum }\end{array}$ & crus I & Nil at $p<0.01$ & Lobule VII, crus I \& II \\
\hline
\end{tabular}




\section{Discussion}

Our study indicates that clinical subtypes of FTD exhibit individual patterns of cerebellar degeneration; these changes are widespread in nfvPPA and bvFTD, but relatively focal in svPPA. Marked cerebellar differences were detected between C9 + ALSFTD and C9-ALSFTD. Our data suggest that certain cerebellar regions, such as lobule V, VI, VIII, vermis, Crus I and II, are more susceptible to degeneration in FTD than other areas. While our findings are in line with previous reports $[14,16]$, one of the novelty of our study is the detection of lobule $\mathrm{V}$ degeneration across the clinical spectrum of FTD. This lobule is part of the anterior cerebellar lobe that primarily mediates sensorimotor functions [4, 41, 42]. However, dichotomising motor and cognitive functions to the anterior and posterior cerebellum may be simplistic; lobule $\mathrm{V}$ is also involved in verbal working memory, emotion and rhythm processing $[3,4]$. This region has previously been implicated in bvFTD cohorts including those with ALSFTD [12]. We have also demonstrated that the cerebellar vermis is involved in nfvPPA, C9-ALSFTD, and to a greater extent in bvFTD. Vermis degeneration has been previously linked to bvFTD and described in ALSFTD [12, 14, 16, 20]. This region is often referred to as the "limbic cerebellum' because of its role in emotion processing and its connectivity with the limbic and paralimbic regions [3]. Structural abnormalities in this region may manifest in a myriad of irregular social or emotional behaviours, including aggression, irritability and disinhibition [11, $43,44]$. A similar constellation of symptoms may occur in opsoclonus myoclonus syndrome, a post-infectious or paraneoplastic disorder that preferentially involves the cerebellar vermis [45]. These observations are further supported by altered cerebello-cerebral connectivity in bipolar affective disorder $[38,46]$. The functional topography of the cerebellum has been gradually elucidated [47] and careful meta-analyses have ascribed specific higher level cognitive functions to distinct cerebellar areas [3, 4]. The affected regions identified in our study within the 'cognitive cerebellum' are involved in emotional processing, attention, executive function, working memory, language including expressive language, and social cognition [3, $4,48]$. Functional MRI studies have confirmed the coactivation of posterior cerebellar and prefrontal cortices during cognitive tasks, patterns which are distinctly different from the activation of the anterior cerebellum and sensorimotor cortices during motor tasks $[5,49,50]$. This pattern of connectivity has been replicated in greater detail in post-mortem studies [51, 52].

We predominantly observed symmetric cerebellar degeneration, with the exception right hemisphere dominant cortical thinning in bvFTD and svPPA. The asymmetric cerebellar findings in svPPA may be linked to the similarly lateralised pathology at a supratentorial level and potentially mediated by crossed cerebellar network [3, $4,14,53,54]$. It is noteworthy, however, that, exclusively left-sided lobule V, crus I-II volume reductions were noted in svPPA on morphometric analyses. These observations highlight that different imaging modalities capture different aspects of cerebellar degeneration. [55]

We detected markedly divergent grey and white matter changes in C9orf72-positive and C9orf72-negative ALSFTD patients. In contrast to the widespread atrophy observed in C9 + ALSFTD, cortical thinning did not reach statistical significance in C9-ALSFTD. This is consistent with the more extensive cerebellar involvement associated with the $C 9$ orf 72 mutation [13, 19, 27, 28]. Cerebellar, cerebral and spinal changes have also been detected in presymptomatic GGGGCC hexanucleotide repeats expansion carriers [56-59]. It is noteworthy, however, that p62-immunoreactive TDP-43-negative neuronal cytoplasmic inclusions were noted in cerebellar granule cells irrespective of $C 9$ orf 72 status [15, 28]. Widespread cerebellar and cerebral degeneration have also been consistently noted in ALS and PLS cohorts without FTD [60-62]. Dysarthria, pseudobulbar affect, and cognitive deficits are commonly observed in ALS, and cerebellar pathology may contribute to these symptoms [63-67]. Interestingly, we detected higher cortical thickness in lobules I-II in C9orf72-negative ALSFTD compared to controls, which may be in line with the proposed compensatory role of the cerebellum in ALS [68-70].

Our findings may have clinical implications. Patients with clinical and genetic FTD subtypes attend a broad range of specialist including neurologist, psychiatrists and medicine for the elderly physicians. Clinical assessments may be heavily weighted towards cognitive and behavioural testing. If a cerebellar exam is performed at all, there is likely to be a greater emphasis on eliciting physical clinical signs. Post-mortem studies that confirmed cerebellar involvement in $C 9$ orf 72 highlighted the absence of overt ante mortem cerebellar signs such as ataxia without considering cognitive manifestations [27, 28]. It is conceivable that a formal cerebellar examination was not performed in some of these cases, and subtle cerebellar deficits may remain unrecognised. Since in our study lobule V degeneration was a consistent finding in all FTD subtypes, and this structure is a principal hub of cerebro-cerebellar sensorimotor networks, we suggest that formal cerebellar examination should be performed in all patients with suspected FTD. In addition, sequencing tasks (visual, verbal, behavioural and spatial) could be considered as a screening tool for cerebellumassociated cognitive dysfunction [6]. In those with apparent autosomal dominant inheritance who test negative for common FTD genes, it is important to consider SCA17; as 
it may initially resemble bvFTD [71]. The establishment of phenotype-specific imaging signatures and biomarker profiles may also aid the accurate categorisation of single subject datasets into relevant diagnostic, phenotypic or prognostic groups [72-76].

In addition to the lack of molecular profiling, a key limitation of our study is the sample size of our cohorts, particularly in those with PPA. Accordingly, our data need to be replicated in larger cohorts and validated by the dedicated assessment of the cerebellum post-mortem. Longitudinal radiological data acquisition may help to further elucidate the dynamic biological processes underpinning the progressive symptoms observed clinically [77]. Future cerebellar studies in FTD may benefit from complementing quantitative MRI analyses with FDG-PET to establish the comparative detection sensitivity of the two modalities. While previous PET studies captured cerebellar hypometabolism, no convergent patterns have been identified [16, 24, 25].

Our own findings, and the limited literature available, suggest that cerebellar degeneration is an important, albeit under investigated facet of FTD research, which merits dedicated clinical, imaging and post-mortem studies. The characterisation of cerebellar pathology in FTD is not merely an academic pursuit. The concomitant degeneration of interconnected infra- and supra-tentorial regions indicates connectivity-mediated propagation mechanisms, which may aid the identification of novel therapeutic targets. The demonstration of markedly divergent cerebellar signatures across the spectrum of FTDs serves as a reminder that FTD is a pathologically heterogeneous condition and the quest for 'one drug for all' is a naïve notion. In line with the principles of precision medicine, phenotype- and genotype-specific disease-modifying strategies are likely offer therapeutic benefits. Pioneering antisense oligonucleotide (ASO) studies in C9orf72 give cause for optimism to target specific genotypes, and coordinated research efforts targeting tau may also pave the way to breakthrough individualised therapies. $[57,78]$ The refinement of clinical screening tools and the development of disease-specific imaging protocols may not only assist the accurate categorisation of suspected FTD patients, but serve as biomarkers in future clinical trials.

\section{Conclusions}

Our data indicate unique cerebellar imaging signatures in FTD phenotypes with the selective involvement of specific lobules. It is conceivable that facets of behavioural and cognitive impairment previously exclusively attributed to supratentorial regions, may in part stem from cerebellar degeneration. Our findings highlight the involvement of infratentorial regions in FTD and support the evolving role of the cerebellum in cognitive and behavioural manifestations.

Acknowledgements Peter Bede and the Computational Neuroimaging Group are supported by the Health Research Board (HRB EIA-2017019), the Irish Institute of Clinical Neuroscience (IICN), the Spastic Paraplegia Foundation (SPF), the EU Joint Programme-Neurodegenerative Disease Research (JPND), the Andrew Lydon scholarship, and the Iris O'Brien Foundation.

Author contribution Drafting the manuscript: $\mathrm{MCM}$ and PB; clinical assessments: MCM, RHC, SLHS, $\mathrm{OH}$, and $\mathrm{SH}$; neuroimaging analyses: MCM, FC, JL, and PB; genetic analyses: MAD, JCH, AV, and RLM; conceptualisation of the study: MCM and PB; revision of the manuscript for intellectual content: MCM, RHC, SLHS, FC, JL, MAD, JCH, $\mathrm{AV}, \mathrm{RLM}, \mathrm{OH}, \mathrm{SH}$, and PB.

Funding Open Access funding provided by the IReL Consortium.

\section{Compliance with ethical standards}

Conflicts of interest The authors of this manuscript have no conflicts of interest to disclose.

Open Access This article is licensed under a Creative Commons Attribution 4.0 International License, which permits use, sharing, adaptation, distribution and reproduction in any medium or format, as long as you give appropriate credit to the original author(s) and the source, provide a link to the Creative Commons licence, and indicate if changes were made. The images or other third party material in this article are included in the article's Creative Commons licence, unless indicated otherwise in a credit line to the material. If material is not included in the article's Creative Commons licence and your intended use is not permitted by statutory regulation or exceeds the permitted use, you will need to obtain permission directly from the copyright holder. To view a copy of this licence, visit http://creativecommons.org/licenses/by/4.0/.

\section{References}

1. Malm J et al (1998) Cognitive impairment in young adults with infratentorial infarcts. Neurology 51(2):433-440

2. Stoodley CJ et al (2016) Location of lesion determines motor vs. cognitive consequences in patients with cerebellar stroke. NeuroImage Clin 12:765-775

3. Keren-Happuch E et al (2014) A meta-analysis of cerebellar contributions to higher cognition from PET and fMRI studies. Hum Brain Mapp 35(2):593-615

4. Stoodley CJ, Schmahmann JD (2009) Functional topography in the human cerebellum: a meta-analysis of neuroimaging studies. Neuroimage 44(2):489-501

5. Argyropoulos GPD et al (2020) The cerebellar cognitive affective/ schmahmann syndrome: a task force paper. Cerebellum (London, England) 19(1):102-125

6. Tedesco AM et al (2011) The cerebellar cognitive profile. Brain 134(12):3672-3686

7. Van Overwalle F, D'Aes T, Marien P (2015) Social cognition and the cerebellum: a meta-analytic connectivity analysis. Hum Brain Mapp 36(12):5137-5154 
8. Runnqvist $E$ et al (2016) Internal modeling of upcoming speech: a causal role of the right posterior cerebellum in non-motor aspects of language production. Cortex 81:203-214

9. Finegan E et al (2019) Pathological crying and laughing in motor neuron disease: pathobiology, screening, Intervention. Front Neurol 10:260

10. Bede P, Finegan E (2018) Revisiting the pathoanatomy of pseudobulbar affect: mechanisms beyond corticobulbar dysfunction. Amyotroph Lateral Scler Frontotemporal Degener 19(1-2):4-6

11. Levisohn L, Cronin-Golomb A, Schmahmann JD (2000) Neuropsychological consequences of cerebellar tumour resection in children: Cerebellar cognitive affective syndrome in a paediatric population. Brain 123(5):1041-1050

12. Tan $\mathrm{R}$ et al (2015) Terra incognita - cerebellar contributions to neuropsychiatric and cognitive dysfunction in behavioral variant frontotemporal dementia. Front Aging Neurosci 7(121)

13. Bocchetta $\mathrm{M}$ et al (2016) Patterns of regional cerebellar atrophy in genetic frontotemporal dementia. Neuroimage Clin 11:287-290

14. Chen $\mathrm{Y}$ et al (2018) Cerebellar atrophy and its contribution to cognition in frontotemporal dementias. Ann Neurol 84(1):98-109

15. Snowden JS et al (2012) Distinct clinical and pathological characteristics of frontotemporal dementia associated with C9ORF72 mutations. Brain 135(Pt 3):693-708

16. Chen $Y$ et al (2019) The cerebellum in frontotemporal dementia: a meta-analysis of neuroimaging studies. Neuropsychol Rev 29(4):450-464

17. Chen $Y$ et al (2020) Cerebellar structural connectivity and contributions to cognition in frontotemporal dementias. Cortex 129:57-67

18. Gellersen HM et al (2017) Cerebellar atrophy in neurodegeneration - a meta-analysis. J Neurol Neurosurg Psychiatry 88(9):780-788

19. Cash DM et al (2018) Patterns of gray matter atrophy in genetic frontotemporal dementia: results from the GENFI study. Neurobiol Aging 62:191-196

20. Tan RH et al (2014) Cerebellar integrity in the amyotrophic lateral sclerosis-frontotemporal dementia continuum. PLoS ONE 9(8):e105632

21. Guo CC et al (2016) Network-selective vulnerability of the human cerebellum to Alzheimer's disease and frontotemporal dementia. Brain J Neurol 139(Pt 5):1527-1538

22. Hardiman O et al (2016) Neurodegenerative Disorders: A Clinical Guide. 2016 ed. 2016, Springer Cham Heidelberg New York Dordrecht London $\odot$ Springer International Publishing Switzerland 2016: Springer International Publishing. 1-336

23. Schmahmann JD (1998) Dysmetria of thought: clinical consequences of cerebellar dysfunction on cognition and affect. Trends Cogn Sci 2(9):362-371

24. Ishii K et al (1998) Cerebral glucose metabolism in patients with frontotemporal dementia. J Nucl Med 39(11):1875-1878

25. Devenney E et al (2014) Frontotemporal dementia associated with the C9ORF72 mutation: a unique clinical profile. JAMA Neurol 71(3):331-339

26. Simón-Sánchez J et al (2012) The clinical and pathological phenotype of C9ORF72 hexanucleotide repeat expansions. Brain 135(Pt 3):723-735

27. Mahoney CJ et al (2012) Frontotemporal dementia with the C9ORF72 hexanucleotide repeat expansion: clinical, neuroanatomical and neuropathological features. Brain J Neurol 135(Pt 3):736-750

28. Whitwell JL et al (2012) Neuroimaging signatures of frontotemporal dementia genetics: C9ORF72, tau, progranulin and sporadics. Brain 135(Pt 3):794-806

29. Ferrer I et al (2015) Familial behavioral variant frontotemporal dementia associated with astrocyte-predominant tauopathy. J Neuropathol Exp Neurol 74(4):370-379
30. Rascovsky K et al (2011) Sensitivity of revised diagnostic criteria for the behavioural variant of frontotemporal dementia. Brain 134:2456-2477

31. Christidi F et al (2019) Hippocampal pathology in amyotrophic lateral sclerosis: selective vulnerability of subfields and their associated projections. Neurobiol Aging 84:178-188

32. Bede $\mathrm{P}$ et al (2019) Brainstem pathology in amyotrophic lateral sclerosis and primary lateral sclerosis: a longitudinal neuroimaging study. Neuroimage Clin 24:102054

33. Chipika RH et al (2020) Amygdala pathology in amyotrophic lateral sclerosis and primary lateral sclerosis. J Neurol Sci 417:117039

34. Bede P et al (2014) Sexual dimorphism in ALS: exploring genderspecific neuroimaging signatures. Amyotroph Lateral Scler Frontotemporal Degener 15(3-4):235-243

35. Diedrichsen J et al (2009) A probabilistic MR atlas of the human cerebellum. Neuroimage 46(1):39-46

36. Manjon JV, Coupe P (2016) volBrain: an online MRI brain volumetry system. Front Neuroinform 10:30

37. Romero JE et al (2017) CERES: a new cerebellum lobule segmentation method. Neuroimage 147:916-924

38. Argyropoulos GD et al (2021) Cerebro-cerebellar white matter connectivity in bipolar disorder and associated polarity subphenotypes. Prog Neuropsychopharmacol Biol Psychiatry 104:110034

39. Finegan E et al (2019) The clinical and radiological profile of primary lateral sclerosis: a population-based study. J Neurol 266(11):2718-2733

40. Chipika RH et al (2020) "Switchboard" malfunction in motor neuron diseases: Selective pathology of thalamic nuclei in amyotrophic lateral sclerosis and primary lateral sclerosis. Neuroimage Clin 27:102300

41. Grodd W et al (2001) Sensorimotor mapping of the human cerebellum: fMRI evidence of somatotopic organization. Hum Brain Mapp 13(2):55-73

42. Bushara KO et al (2001) Multiple tactile maps in the human cerebellum. NeuroReport 12(11):2483-2486

43. Tavano A et al (2007) Disorders of cognitive and affective development in cerebellar malformations. Brain $130(\mathrm{Pt}$ 10):2646-2660

44. Lupo M et al (2018) Development of a psychiatric disorder linked to cerebellar lesions. Cerebellum 17(4):438-446

45. Turkel SB et al (2006) Mood and behavioral dysfunction with opsoclonus-myoclonus ataxia. J Neuropsychiatry Clin Neurosci 18(2):239-241

46. Strakowski SM, Delbello MP, Adler CM (2005) The functional neuroanatomy of bipolar disorder: a review of neuroimaging findings. Mol Psychiatry 10(1):105-116

47. Bernard JA, Mittal VA (2014) Cerebellar-motor dysfunction in schizophrenia and psychosis-risk: the importance of regional cerebellar analysis approaches. Front Psychiatry 5:160

48. Brissenden JA et al (2016) Functional evidence for a cerebellar node of the dorsal attention network. J Neurosci 36(22):6083-6096

49. Buckner RL et al (2011) The organization of the human cerebellum estimated by intrinsic functional connectivity. J Neurophysiol 106(5):2322-2345

50. Palesi F et al (2017) Contralateral cortico-ponto-cerebellar pathways reconstruction in humans in vivo: implications for reciprocal cerebro-cerebellar structural connectivity in motor and non-motor areas. Sci Rep 7(1):12841

51. Takahashi E et al (2013) Detection of postmortem human cerebellar cortex and white matter pathways using high angular resolution diffusion tractography: a feasibility study. Neuroimage 68:105-111 
52. Steele CJ et al (2017) Human cerebellar sub-millimeter diffusion imaging reveals the motor and non-motor topography of the dentate nucleus. Cereb Cortex 27(9):4537-4548

53. Bede P et al (2018) Connectivity-based characterisation of subcortical grey matter pathology in frontotemporal dementia and ALS: a multimodal neuroimaging study. Brain Imaging Behav 12(6):1696-1707

54. Omer T et al (2017) Neuroimaging patterns along the ALS-FTD spectrum: a multiparametric imaging study. Amyotroph Lateral Scler Frontotemporal Degener 18(7-8):611-623

55. Nasseroleslami B et al (2019) Characteristic increases in EEG connectivity correlate with changes of structural MRI in amyotrophic lateral sclerosis. Cereb Cortex 29(1):27-41

56. Rohrer JD et al (2015) Presymptomatic cognitive and neuroanatomical changes in genetic frontotemporal dementia in the Genetic Frontotemporal dementia Initiative (GENFI) study: a cross-sectional analysis. Lancet Neurol 14(3):253-262

57. Li Hi Shing S et al (2021) The imaging signature of C9orf72 hexanucleotide repeat expansions: implications for clinical trials and therapy development. Brain Imaging Behav

58. Querin G et al (2019) Presymptomatic spinal cord pathology in c9orf72 mutation carriers: a longitudinal neuroimaging study. Ann Neurol 86(2):158-167

59. El Mendili MM et al (2019) Spinal cord imaging in amyotrophic lateral sclerosis: historical concepts-novel techniques. Front Neurol 10:350

60. Finegan E et al (2021) Extra-motor cerebral changes and manifestations in primary lateral sclerosis. Brain Imaging Behav

61. Dukic $S$ et al (2019) Patterned functional network disruption in amyotrophic lateral sclerosis. Hum Brain Mapp 40(16):4827-4842

62. Finegan E et al (2019) Primary lateral sclerosis: a distinct entity or part of the ALS spectrum? Amyotroph Lateral Scler Frontotemporal Degener 20(3-4):133-145

63. Yunusova $Y$ et al (2019) Clinical measures of bulbar dysfunction in ALS. Front Neurol 10:106

64. Christidi F et al (2018) Clinical and radiological markers of extra-motor deficits in amyotrophic lateral sclerosis. Front Neurol 9:1005

65. Burke $\mathrm{T}$ et al (2016) Measurement of social cognition in amyotrophic lateral sclerosis: a population based study. PLoS ONE 11(8):e0160850
66. Burke T et al (2016) Discordant performance on the 'Reading the Mind in the Eyes' Test, based on disease onset in amyotrophic lateral sclerosis. Amyotroph Lateral Scler Frontotemporal Degener 17(7-8):467-472

67. Verstraete E et al (2015) Mind the gap: the mismatch between clinical and imaging metrics in ALS. Amyotroph Lateral Scler Frontotemporal Degener 16(7-8):524-529

68. Abidi $\mathrm{M}$ et al (2020) Adaptive functional reorganization in amyotrophic lateral sclerosis: coexisting degenerative and compensatory changes. Eur J Neurol 27(1):121-128

69. Abidi M et al (2020) Neural correlates of motor imagery of gait in amyotrophic lateral sclerosis. J Magn Reson Imaging 53(1):223-233

70. Proudfoot M, Bede P, Turner MR (2018) Imaging cerebral activity in amyotrophic lateral sclerosis. Front Neurol 9:1148

71. Rolfs A et al (2003) Clinical features and neuropathology of autosomal dominant spinocerebellar ataxia (SCA17). Ann Neurol 54(3):367-375

72. Grollemund V et al (2019) Machine learning in amyotrophic lateral sclerosis: achievements, pitfalls, and future directions. Front Neurosci 13:135

73. Devos D et al (2019) A ferroptosis-based panel of prognostic biomarkers for amyotrophic lateral sclerosis. Sci Rep 9(1):2918

74. Querin G et al (2018) Multimodal spinal cord MRI offers accurate diagnostic classification in ALS. J Neurol Neurosurg Psychiatry 89(11):1220-1221

75. Blasco $\mathrm{H}$ et al (2018) A pharmaco-metabolomics approach in a clinical trial of ALS: identification of predictive markers of progression. PLoS ONE 13(6): 0198116

76. Tahedl $\mathrm{M}$ et al (2021) Cortical progression patterns in individual ALS patients across multiple timepoints: a mosaic-based approach for clinical use. J Neurol 268(5):1913-1926

77. Chipika RH et al (2019) Tracking a fast-moving disease: longitudinal markers, monitoring, and clinical trial endpoints in ALS. Front Neurol 10:229

78. Panza F et al (2020) Development of disease-modifying drugs for frontotemporal dementia spectrum disorders. Nat Rev Neurol 16(4):213-228 